\title{
Chiron and the Centaurs: escapees from
} the Kuiper belt

\author{
Alan Stern \& Humberto Campins
}

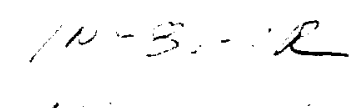

The Centaurs - a group of objects orbiting chaotically among the giant planets of our Solar System - appear to be a population transitional in size between typical short-period comets and the large Kuiper-belt objects that orbit beyond Neptune. They promise to reveal much about the origin of and interrelationships between the icy bodies of the outer Solar System.

THE outer Solar System his long appeared to be a largely empty place, inhabited only by the four giant planets, Pluto and a transient population of comets. In 1977 however, a faint and enigmatic object - 2060 Chiron-was discovered' moving on a moderately inclined, strongly chaotic 51 -year orbit which takes it from just inside Saturn's orbit out almost as far as that of Uranus. It was not initially clear from where Chiron originated.

Following Chiron's discovery, almost 15 years elapsed before other similar objects were discovered; five more have now been ideritified'. Based on the detection statistics implied by these discoveries, it has become clear that these objects belong to a significant population of several hundred (or possibly several thousand) large icy bodies moving on relatively short-lived orbits between the giant planets. This new class of objects, known collectively as the Centaurs, are intermediate in diameter between typical comets $(1-20 \mathrm{~km})$ and small icy planets such as Pluto $(\sim 2,300 \mathrm{~km})$ and Triton $(\sim 2,700 \mathrm{~km})$. Although the Centaurs are interesting in their own right, they have taken on added significance following the recognition that they most probably originated in the ancient reservoir of comets and larger objects located beyond the orbit of Neptune known as the Kuiper belt.

\section{Origin of the Centaurs}

The first clue to the origin of the Centaurs came about as a result of dynamical studies of Chiron's orbit. At first discovered in the late $1970 \mathrm{~s}^{3}$, and forcefully reiterated in more modern calculations ${ }^{4}$, Chiron's orbit is highly unstable to perturbations by the giant planets. As a result, Chiron's orbital lifetime among the giant planets is short, leading to the conclusion that its origin was in a more stable reservoir, either in the asteroid belt, or beyond the giant planets. The discovery of a coma around Chiron ${ }^{5-7}$, in the late 1980s, indicated the presence of surface volatiles which could not have survived the age of the Solar System in the comparatively warm asteroid belt ${ }^{\text {s; }}$ such volatiles therefore strongly indicate that Chiron originated in a distant reservoir, beyond the giant planets.

A second line of evidence relating to Chiron's origin came about from simulations of cometary dynamics. These studies ${ }^{9-12}$ demonstrated that the dominant dynamical class of short-period comets, called the Jupiter-family comets (JFC) cannot be derived from the classical, Oort-cloud cometary reservoir. The reason for this is that their characteristically low orbital inclinations cannot be efficiently produced by the action of planetary perturbations on orbits initially in the inclination-randomized (that is, nearly spherical) Oort cloud.

Instead, the JFC $\operatorname{secm}$ to derive from a dynamically stable reservoir concentrated nein the plane of the planetany system. Any such reservoin for the HI: must satisfy the criterion that the loss rate of objects from it he low ensugh that the reservoir can persist for the age of the Solar System. Because the dynamical clearing time for on hits hetweon lhe planets is characteristically one to lwo orders of mignmude shomler than lle age of the Solar System? 14, there are low regions of spate that provide stable,

candidate source locations for the JFC. One such region is the zone beyond Neptune's orbit (at $30 \mathrm{AU}$, astronomical units) where nonlinear perturbations by Neptune and the other giant planets can excite orbital eccentricities on timescales comparable to the age of the Solar System. Once orbital eccentricities are excited sufficiently to cause objects to cross Neptune's orbit, a fraction of these objects become temporarily trapped on Centaur-like orbits among the giant planets. Other such objects are dynamically transported by subsequent encounters with the other giant planets onto orbits that pass within 1-2 AU of the Sun ${ }^{15}$, where they generate comae and become easily detectable. A second possible source region for the JFC is the slowly dynamically evaporating jovian Trojan clouds, whose dynamics are controlled by the stability of a narrow phase space surrounding the leading and trailing lagrangian points of Jupiter. However, recent dynamical simulations $^{16}$ show that the jovian Trojan clouds are not as effective as the so-called trans-neptunian zone in populating the JFC and Centaur populations. Following historical suggestions dating back as far as the 1940s that a disk-shaped reservoir of planetesimals and other small objects might reside beyond the Neptune ${ }^{17.18}$, the trans-neptunian region has been dubbed the Kuiper belt, or in analogy to debris belts around the other stars, the Kuiper disk.

The pivotal breakthrough concerning the reality of the hypothesized Kuiper belt came in 1992, with the discovery of a faint ( $R$ band magnitude near 23 ), $180-\mathrm{km}$ diameter object ${ }^{19}$ designated $1992 \mathrm{QB}_{1} .1992 \mathrm{QB}$, orbits the Sun in a stable, nearly circular orbit some $14 \mathrm{AU}$ beyond Neptune. In the four years since $1992 \mathrm{QB}_{1}$ was found, over three dozen similar objects have been discovered in the trans-neptunian region ${ }^{2}$. Estimates ${ }^{2,20}$ indicate that some of these objects have diameters approaching $400 \mathrm{~km}$. Based on the efficiency with which such objects are being detected and their surface density on the sky, it has been estimated that around $7 \times 10^{4}$ objects with diameters greater than $100 \mathrm{~km}$ orbit between 30 and $50 \mathrm{Au}$ (ref. 2). Here we refer to these larger objects populating the Kuiper Belt as $\mathrm{QB}_{1} \mathrm{~s}$.

Following the discoveries of numerous $\mathrm{QB}_{1} \mathrm{~s}$ in the Kuiper belt, the Hubble Space Telescope (HST) was used to conduct a search for the much smaller, and much fainter, cometary nuclei which must be present in this region if it is indeed a source of the JFCs. Last year, Cochran et al. ${ }^{21}$ reported exciting evidence, near the limit of HST's capabilities, for numerous objects with V-band magniludes of $\sim 28.6$, corresponding to comet-like diameters of a few kilometres to $\sim 10 \mathrm{~km}$. This evidence corresponds to a population of several hundred million comet-sized objects. If this result is coupled with models' ${ }^{12}$ that predict the ratio of the population detected in the region searched by Cochtan to the entire transneplunian rone, a lotal population is calculated of several billion comets in the 30-50 Au region. As such, it appears that the Kuiper bett is indeed the source region of most Jl:C, as dymamical simulations predicted ${ }^{9.14}$

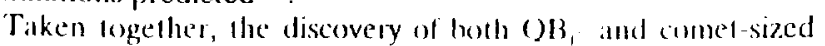



objects in the Kuiper-belt region indicates that the Kuipes belt supplies a wide size range of objects onto onthis an the giantplaned region. Basted both on expectations resulting from the planetesimal accretion codes, and the observational evidence for many more comets than $\mathrm{QB}_{1} \mathrm{~s}$, it appears that a powerlaw-like source population histogramm exists in the Kuiper belt, with many more small bodies than $\mathrm{QB}_{1} \mathrm{~s}$. Because the dynamical transport process that brings objects from the belt to planet-crossing orbits is essentially independent of the mass of the object being transported ${ }^{15}$, it is expected that the population of objects ranging in size from Centaurs down to JFC orbiting among the giant planets is representative of the population of objects in the 30-50 Au zone from which they are derived.

\section{Physical attributes of the Centaurs}

It is now established that the slow leakage of objects from the Kuiper belt due to planetary perturbations creates a population of objects on comparatively short-lived, planet-crossing orbits in the giant-planet region between 5 and $30 \mathrm{Au}$ from the Sun. Studies of the dynamical evolution of orbits dislodged from the Kuiper belt ${ }^{15}$ predict a characteristic equilibrium population of objects on planet-crossing orbits that is $\sim 10^{-4}$ of the population of the Kuiper-belt reservoir from which they are derived. These studies also predict that the median lifetime of such orbits is of the order of $5 \times 10^{7}$ years. Such findings imply that a population of $\sim 5 \times 10^{5}$ to perhaps $10^{6}$ comets, and $\sim 30-300$ Centaur objects of diameter $100 \mathrm{~km}$ or larger, are orbiting between the giant planets.

Chiron and its recently discovered cohort of Centaurs are thus now seen to be objects derived from the Kuiper belt. Table 1 summarizes the orbital attributes of the six known Centaurs; Fig. 1 depicts the orbits of these objects and their dynamical context in the outer Solar System.

As escapees from the Kuiper belt, the Centaurs are an important population for study. Indeed, owing to their greater proximity to the Sun, the brightest centaurs are some 5-7 astronomical magnitudes (factors of $\sim 100$ to $\sim 600$ ) brighter than the brightest

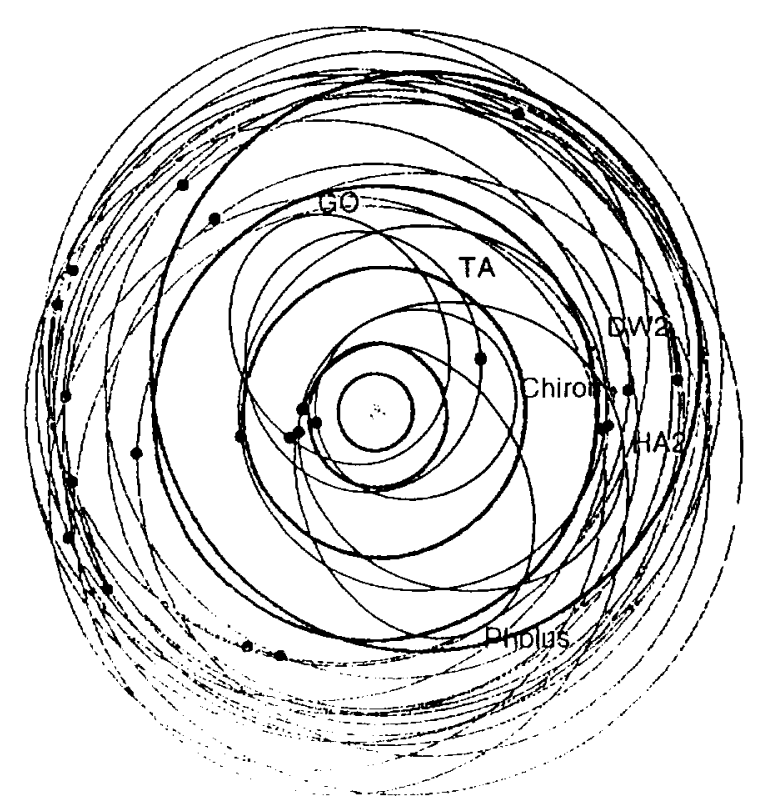

Kuiper-belt objects, which enables more detailed studies of the Centaurs than are possible with the $\mathrm{QB}, \mathrm{s}$ and Kuiper-belt comets. Additionally, being closer to the Sun, the Centaurs experience greatel heating, which generates characteristic perihelion surface temperatures in the range $\sim 120$ to $150 \mathrm{~K}$ (ref. 22 ); by contrast, Kuiper-belt objects probably never experience surface temperatures in excess of $60-70 \mathrm{~K}$. Therefore, because vapour pressure depends exponentially on the temperature of the ice, Centaurs are much more likely than Kuiper-belt objects to show sublimationgenerated activity. Although such heating causes the surfaces of the Centaurs to evolve chemically and physically over long timescales ${ }^{23}$, it also causes the surface ices to sublime, and thus reveal valuable insights into the nature of these objects.

Unfortunately, although the Centaurs are brighter than Kuiperbelt objects, they are still faint in absolute terms, so considerable dedication is required to obtain physical information on them. As a result, comparatively little work has been done to reveal their compositions, colours, shapes, rotational properties and other attributes (Table 2). Despite the great deficits in our knowledge about the physical and chemical characteristics of this unique population, several important pieces of information are emerging.

First, with regard to the derived sizes of the Centaurs discovered to date, roughly half appear to be near $60 \mathrm{~km}$ in diameter, but 2060 Chiron $^{22.24}$ and 5145 Pholus $^{25}$ are much larger, with $\sim 180-\mathrm{km}$ diameters that are comparable to typical $\mathrm{QB}_{1} \mathrm{~s}$ being discovered in the trans-neptunian zone. Second, infrared spectroscopy and colour photometry have given the first clues about the surface compositions of these objects. The first clearly detected spectral

FIG. 1 The orbits of the giant planets (black lines), the six known Centaurs (red lines) and those Kuiper-belt objects with well established orbits (green lines). The dot on each orbit depicts the current location of the object. For scale, Jupiter's orbit is approximately $10 \mathrm{Au}$ across. Abbreviations on the figure as follows: GO 1995GO; TA, 1994TA; DW2, 1995DW2; Chiron, 2060 Chiron; HA2, 1993HA 2 ; Pholus, 5145 Pholus. (Figure courtesy of H. F. Levison) 

TABIE 2 Physical characteristics of the known Centaurs

\begin{tabular}{|c|c|c|c|c|c|c|}
\hline Objest & $\begin{array}{c}\text { Diameter } \\
(\mathrm{km})\end{array}$ & $\begin{array}{c}\text { Geometric } \\
\text { albedo (in V) }\end{array}$ & $\begin{array}{l}\text { Rotalionial } \\
\text { period }\end{array}$ & $\begin{array}{l}\text { Rotatıonal } \\
\text { amplitude }\end{array}$ & $v$ / colomirt & $\begin{array}{l}\text { Detected } \\
\text { activity }\end{array}$ \\
\hline 2060 Chıron & $182+10$ & $0.11-0.20$ & $5.92 \mathrm{~h}$ & $9 \%$ & 1131004 & Yes \\
\hline 51.45 Pholus & 1851.22 & 0.0410 .02 & $9.98 \mathrm{~h}$ & $20 \%$ & $253+006$ & No \\
\hline $1993 \mathrm{HA}_{2}$ & $62 \mathrm{kmt}$ & 0.05 & & & $207+040$ & No \\
\hline $1994 T A^{2}$ & $28 \mathrm{~km} 1$ & 0.05 & & & & No \\
\hline 1995DW2 & $68 \mathrm{kmt}$ & 0.05 & & & & No \\
\hline $1995 \mathrm{GO}$ & $60 \mathrm{kmt}$ & 0.05 & & & & No \\
\hline
\end{tabular}

* The sizes of Chiron and Pholus were obtained 22.24 .25 from thermal fluxes, with computed albedos based on their sizes and $V$ magnitudes. Chiron's albedo is strictly an upper limit owing to a possible, small, residual coma contribution.

† These sizes were computed by assuming a $V$-band geometric albedo of $5 \%$, a value which is commonly found for cometary surfaces.

$\ddagger$ Colour data are discussed in the $1 \mathrm{ext}^{27.31 .32}$. Nole a $V-J$ colour of 1.116 would be identical to the Sun ${ }^{47}$, thereby indicating a neutrally coloured surface. Higher $V-J$ colours indicate red surfaces.

absorption feature among the Centaurs was a deep $2.25-\mu \mathrm{m}$ absorption on Pholus ${ }^{26}$. This feature has been associated with lighı organic solids mixed with ices ${ }^{27,28}$. Importantly a $2.04-\mu \mathrm{m}$ absorption features has also now been detected, both in Pholus and Chiron ${ }^{29}$, which Cruikshank et al. identify ${ }^{30}$ in Chiron as an absorption band of water-ice. Third, although none of the three Centaurs that have been explored in the infrared have displayed any statistically significant colour variation with rotational phase ${ }^{31}$ they do show striking colour differences between one another (refs 27, 32 and Fig. 2). Indeed, whereas Chiron's intrinsic colour is grey (that is, neutral) throughout the $0.3-2.5 \mu \mathrm{m}$ band, Pholus, which lies in a similar orbit and is similar in size, is extremely red. $1993 \mathrm{HA}_{2}$, though smaller and in a more distant orbit, is also very red compared to Chiron. How much of these differences between various Centaurs is due to evolutionary mechanisms (as opposed to intrinsic attributes) is not yet clear, but it is well established that long-term exposure to cosmic rays and solar ultraviolet radiation darkens (and initially reddens) surfaces containing light-weight organics, in turn creating a more complex chemical mélange.

Additionally, the determination of well constrained albedos for 2060 Chiron, and more particularly 5145 Pholus (because it is not active), provides useful information for predicting the sizes of $\mathrm{QB}_{1} \mathrm{~s}$ from their observed magnitudes.

\section{The particular value of Chiron}

Chiron is uniquely valuable among the Centaurs because of the long history of its sporadic outbursts. Why has such activity only been detected in Chiron? Possibly Chiron is dynamically younger, and therefore more active than the other objects. Alternatively the other objects may have thicker surface mantles, may be fundamentally different in their composition or simply may have not been observed long enough to expect to detect activity. Chiron's

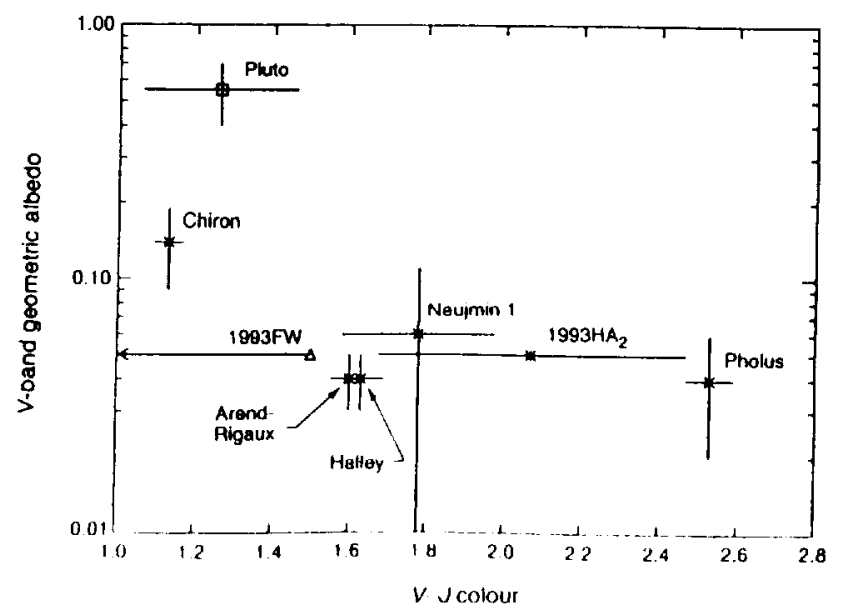

uniqueness in showing activity is perlaps the most intriguing observable obtained on the Centaurs so far.

Chiron's activity was first recognized when it suffered an outburst that increased its brightness by a factor (in 1989) of just over two ${ }^{33.34}$. In 1989-91 Chiron was also observed to show a highly variable particulate coma and tail extending as far as $2 \times 10^{6} \mathrm{~km}$ (refs 35,36 ), and a cloud of $\mathrm{CN}$ gas ${ }^{37}$ presumably derived from the photodissociation of some heavier, parent molecule. When these various observations were made, Chiron was still more than $10 \mathrm{AU}$ from the Sun, where the solar radiation field is too weak to sublime water-ice, the common volatile that powers the cometary activity close to the Sun. Although other mechanisms remain plausible, the sublimation of highly volatile ices like $\mathrm{CO}, \mathrm{N}_{2}$ or $\mathrm{CH}_{4}$ (buried a short distance below the surface) were therefore flavoured as the source of Chiron's activity. Further evidence for the sublimation of such volatiles was obtained through the discovery of even more extreme activity on archival, pre-discovery images of Chiron obtained when it was near its aphelion at $19 \mathrm{AU}$, and therefore far too cold to sublime anything but highly volatile ices like those mentioned above ${ }^{38}$. The final confirmation of this hypothesis came in 1995, though the discovery of CO gas itself in Chiron's coma ${ }^{39,40}$.

The fact that Chiron's activity was greater at its aphelion than it has been at any time since provides compelling evidence that its level of activity is not a simple function of heliocentric distance alone. Instead, Chiron's activity probably involves a complex interaction between the level of insolation reaching its surface, the obliquity of its spin axis, the location of its near-surface volatiles and extensive surface mantling by substances (possibly including silicates, water-ice and carbonaceous materials) which do not strongly sublime that far from the Sun.

Chiron's strong variability and the low gas-production rate inferred from $\mathrm{CN}$ and $\mathrm{CO}$ gas detections in its coma provide
FIG. 2 Colour-albedo diagram showing the visible-band albedos and visible-infrared (that is, $V-J$ ) colours for several Centaurs (Chiron, Pholus and $1993\left(\mathrm{~A}_{2}\right.$ ), several cometary nuclei (Arend-Rigaux, Halley and Neujmin 1). Pluto and the one $\mathrm{QB}_{1}$ (1993FW) for which applicable data are available. A neutrally coloured surface would have the $V-J$ colour of the Sun, 1.116 (ref. 54). Note that the Centaur Pholus is quite red; in fact, it is far redder than any other object in the Solar System. The $V-J$ colour for 1993FW is an upper limit. $V-J$ for Pluto was obtained form D. P. Cruikshank (personal communication; the $V$-albedo error bars for Pluto represent its intrinsic rotational lightcurve variation. 

siong cyidence that Chron's artivily is generated by localized strong cvidence that ( hources covering $61 \%$ of the surface The case supporting highly localized vents on jets on the surface is further supported by shortterm brightness fluctuations in ('monts comat ${ }^{41}$ that occur on timescales consistent with clouts of material being ejected onto suborbital trajectories, and by the detection of complex opacity structures in Chron's coma duing two recently observed stellar occulations by Chiron ${ }^{24,43}$. I1 has been pointed out ${ }^{44}$ that these vents or jets may resemble the geysers detected on the surface of Neptune's large, captured satellite, Triton.

Chiron's low gravity creates a situation in which its escape speed $\left(\sim 10^{2} \mathrm{~m} \mathrm{~s}^{-1}\right)$ is comparable to both the thermal velocity of subliming gas $\left(\sim 2 \times 10^{2} \mathrm{~m} \mathrm{~s}^{-1}\right)$, and the estimated muzzle velocity of Triton-like geysers $\mathrm{s}^{4.5}$ (of the order of $40-300 \mathrm{~m} \mathrm{~s}^{-1}$ ). As a result, some of the gas and entrained particulates ejected from Chiron's surface would be deposited into high suborbital trajectories; much would also escape. Modellers have only begun to explore the range of interesting physical phenomena likely to result in this intermediate regime between frecly escaping cometary comae and strongly bound planetary atmospheres ${ }^{46}$. Among these is the distinct possibility that Chiron's neutral colour and comparatively high albedo are the direct result of its activity, which probably causes a thin veneer of icy particulates to rain back onto the surface from suborbital trajectories.

\section{An emerging view}

We are witnessing a revolution in our understanding of the content and architecture of the outer Solar System. Whereas a decade ago, the outer Solar System seemed to consist only of the outer planets, the Oort-cloud comets and the then-rogue object Chiron, we now see revealed both the teeming Kuiper belt and its progeny, the comets and Centaur-sized objects orbiting among the outer planets. As a result, we have come to recognize that the

1. Kowal, C. T. tcarus 77, 118-123 (1989)

1. Weissman, P. R. \& Levison, H. F. in Pluto \& Charon (eds Tholen, D. J. \& Stern, S. A) (Univ. Arizona Press, Tucson, in the press).

3. Oikawa S. Evernart. E. Astron. J. 84, 134-139 (1979)

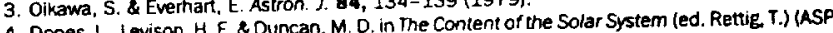
Conf. Ser., in the press).

Cont. Ser., In the press).

5. Tholen, D. J. et al. LAU Circ. No. 4554 (1988).

6. Hartmann, W. K., Cruikshank, D. P. \& Tholen, D. J. Icarus 83, 1-15

7. Meech, K. J Eelton, M. Soc. Pacif. 101, 126-132 (1989).

8. Stem, S. A. Pub. Astron. So Tremaine, S. 328, L69-L73 (1988)

9. Ouncan, M. J., Quinn, T. \& Tremaincan. M. J. Astrophys. J. 355, 667-679 (1990)

10. Quinn, I. R.,

11. Levison, H. F. \& Duncan, M. J. Astrophys. J. 406, L 110, 3073-3081 (1995)

12. Duncan, M. J., Levison, H. F. a Budd, S. M. Ast

13. Gladman, B. \& Duncan, M. J. Astron. J. 100, 1680-1693 (1990).

14. Wisdom, J. \& Hoiman, M. Astron. J. 102, 1528-1538

15. Levison, H. F. Duncan, M. J. Kcarus (submitted).

16. Levison, H. F. \& Shoemaker, E. Icarus (submitted). New York, 1951)

Not. R. Astron. Soc. 109, 600-609 (1949).

18. Edew

19. Jewitt, D. C. \& LuU, J. X. Nature 362, 730-732 1893 (1995)

21. Cochran, A. L. et al. Astrophys. J. 455, 342-346 (1995).

22. Campins, H. et al. Astron. J. 108, 2318-2322 (1994).

23. Mumma, M. J. Weissman, P. R. \& Stern, S. A. in Protostars and P

24. Bus, S. J. et al. Karus (in the press).

25. Bus, S. Ket al laU Circ No 5698 (1996)

25. Davies, K. el all IA Che No 166-169 (1993)

26. Daves, J. Kr al Icas 102, Greenberg, M.) (1994).

27. Crulkshank, D. P. In Inemallonar School a Space Chemiscy (ed. Greenbers

28. Wilson, P. D., Sagan, C. \& Inompson, W. R. Icarus 107, 288-303 (19

29. Luw, I X. Jewitt, D. C. \& Cloutus, E. icarus

31. Buie. M. W. \& Bus, S. J. karus 100, 288.294 (1992) outer Solar System is littered with icy objects intemediate in size belween comets and the glan planets.

We have also leaned that the carly stages of planetary formaIion, with widespread growth from planelesimals to objects with dianeters of several hundreds of kilometres, provide concrete evrtence for an ancient era of planet-building in the Kuiper-belt region ${ }^{424 x}$. For some reason (probably involving the role of Neptune that excited orbital eccentricities that were not conducive 10 (urther growth), the era of accretion in the Kuiper belt was prematurely trunctated at a stage where intermediate-sized objects had formed ${ }^{49}$. The strong circumstantial evidence for the early formation of numerous objects in the $1,000-\mathrm{km}$ class, of which Pluto and Triton are apparently the sole extant remnants within observational reach ${ }^{3151}$, further supports the case for initially strong but eventually arrested planetary accretion in the Kuiper-belt Iegion ${ }^{48.52}$. As such, the Kuiper belt has become one of the most important regions in the Solar System for studies of planetary origins. The Centaurs and $\mathrm{QB}_{1}$ s therefore represent a valuable, relic population of icy objects whose growth was arrested at a fascinating, intermediate stage between comets and small planets.

The Centaurs also serve as bright proxies for distant comets, as laboratories for studying sulface processes occurring on comets, Triton and perhaps Pluto, and as nearby proxies for the $\mathrm{QB}_{1} \mathrm{~s}$ and other intermediate-scale bodies that bridge the size gap between comets, Pluto and Triton. As such, they hold special promise for understanding the origin and interrelationships among the icy bodies of the outer Solar System.

A. Stern is at the Space Science Department, Southwest Research Institute, Boulder, Colorado 80302, USA. H. Campins is at the Department of Astronomy, University of Florida, Gainesville, Florida 32611, USA.

32. Oaves, J. $K$ Tholen, D. J. in The Content of the Solar System led. Reetig. T.) (ASP Cont. Ser., in the press).

The press).

33. Tholen, $D$. J. et ar. AMU Crc. No. 45s4 (1988). J. Jarus B3, 1-15 (1989)

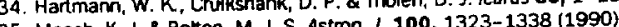

36. Larson, 5. Marcialis, R. L. LAU Cre. No. 5669 (1992).

36. Larson, S. \& Marcialis, R. L. LAU Orc. No. S669.
37. Bus, S. J. Science 251, 774-776 (1991).

38. Bus, S. J. et al. in Proc. Distant Comet Activity Workshop (eds Keller, H. U.\& Huebner, W.) 41-43 (Southwest Res. Inst. San Antonio, Texas, 1993).

39. Womack, M. \& Stern, S. A. LAU Crc. No. 6193 (1995).

40. Womack, M. Stem, S. A. Astrophys. J. (submitted).

41. Wor $x$. A. 100, 913-932 (1990)

41. Lu, J. X Jewn, D. C. A. A

42. Buratti, B. J. \& Dunbar, R. S. Astrophys. J65)

43. Elliot, J. L et al. Nature 373, 46-48 (1995).

44. Stern, S. A., Jackson, A. A. \& Boice, D. C. Astron. J. 107, 765-771 (1994).

45. Kirk, $R$.

1995). 134-139 (Southwest Res. Inst., San Antonio, Texas, 1993).

7. Stern, S. A. Astron. J. (in the press).

48. Davis, D. A. \& Farinetla, P. Icans (submitted).

48. Davis, D. R. \& Faninetla, P. Ican's (submitted).

49. Stern, S. A. Astron. J. 110, 856-865 (1995).

50. Mckinnon, W. B. Nature 311, 355-358
51. Stern, S. A. Icarus $90,271-281$ (1991).

5tem. S. A. McKinnon, W. B. \& Lunine, J.1, in Plulo \& Charon (eds Tholen, 0. J. \& Stem, S. A.) (Unin. Arizona Press, Tucson, in the press)

53. Marsden B. \& Williams, G. W. Minar Planet Electronic Circular, 1996-E02, 1996.

54. Campins, H., Rieke, G. H. \& Lebofsky, M. J. Astron. J. 90, 896-899 (1985) ACKNOWLEDGEMENTS. We thank our colleagues D. Cruikshank, D. Jewitt, S. Larson, H. Levison, R. Marcialis, B. Marsden, W. Mckinnor
A. Fiusimmons for a helpful review.

CORRESPONDENCE should be addressed to A.S. (e-mail: astern@swri.edu) 
